\title{
Práticas de cuidado em saúde mental desenvolvidas por enfermeiros na Estratégia Saúde da Família
}

RESUMO | Objetiva-se compreender as práticas de cuidado em saúde mental desenvolvidas por enfermeiros no âmbito da Estratégia Saúde da Família. Método: pesquisa exploratória, de abordagem qualitativa. Participaram do estudo 10 enfermeiros atuantes nas Estratégias Saúde da Família de um município da região Centro-Sul do Estado do Ceará. Resultados: a partir dos dados emergiram as categorias: Compreensão dos enfermeiros sobre o cuidado em saúde mental na Estratégia Saúde da Família e Práticas do enfermeiro no cuidado à Saúde Mental na Estratégia Saúde da Família. Conclusão: os enfermeiros não se sentem preparados para trabalhar com saúde mental, culminando na oferta de ações superficiais, sem considerar as necessidades reais dos usuários. É apontado, mesmo que poucas vezes, o acolhimento, a escuta, a preocupação com o retorno dos usuários as unidades e o referenciamento dos pacientes para os serviços especializados.

Palavras-chaves: Enfermagem; Estratégia Saúde da Família; Saúde Mental.

\begin{abstract}
It aims to understand the mental health care practices developed by nurses within the scope of the Family Health Strategy. Method: exploratory, qualitative approach. A total of 10 nurses working in the Family Health Strategies of a municipality in the Center-South region of the State of Ceará participated in the study. Results: from the data emerged the categories: Nurses' understanding of mental health care in Family Health Strategies and Nurses Practices in Mental Health Care in Family Health Strategies. Conclusion: nurses do not feel prepared to work with mental health, culminating in the offer of superficial actions, without considering the real needs of the users. It is pointed out, even rarely, the reception, listening, concern with the return of users to the units and referencing of patients for specialized services.
\end{abstract}

Keywords: Nursing; Family Health Strategy; Mental health.

RESUMEN | Su objetivo es comprender las prácticas de atención de salud mental desarrolladas por las enfermeras en el ámbito de la Estrategia de Salud Familiar. Método: exploratorio, enfoque cualitativo. Un total de 10 enfermeras que trabajan en las Estrategias de Salud Familiar de un municipio en la región Centro-Sur del estado de Ceará participaron en el estudio. Resultados: de los datos surgieron las categorías: comprensión de las enfermeras sobre la atención de salud mental en estrategias de salud familiar y prácticas de enfermería en la atención de salud mental en estrategias de salud familiar. Conclusión: las enfermeras no se sienten preparadas para trabajar con la salud mental, culminando en la oferta de acciones superficiales, sin considerar las necesidades reales de los usuarios. Se señala, incluso en raras ocasiones, la recepción, la escucha, la preocupación por el regreso de los usuarios a las unidades y la referencia de pacientes a servicios especializados.

Palavras claves: Enfermería; Estrategia Salud de la Familia; Salud mental.

\section{José Adelmo da Silva Filho}

Enfermeiro. Mestrando em Enfermagem pela Universidade Regional do Cariri. Especialista em Saúde Mental Coletiva pela Escola de Saúde Pública do Ceará.

\section{Cicero Rafael Lopes da Silva}

Enfermeiro. Especialista em Enfermagem Dermatológica pelas Faculdades Integradas de Patos - FIP. Professor do Departamento de Enfermagem da Faculdade de Juazeiro do Norte - FJN.

\section{Anna Polianna Batista Ferreira Marques}

Enfermeira. Residente em Saúde Mental Coletiva pela Escola de Saúde Pública do Ceará.

\section{Riani Joyce Neves Nóbrega}

Enfermeira. Mestre em Saúde da Criança e do Adolescente pela Universidade Estadual do Ceará. Professora do Departamento de Enfermagem da Universidade Regional do Cariri.

\section{Antônio Germane Alves Pinto}

Enfermeiro. Doutor em Saúde Coletiva pela Universidade Estadual do Ceará. Professor Adjunto K do Departamento de Enfermagem da Universidade Regional do Cariri.

Recebido em: 14/07/2019

Aprovado em: 17/12/2019

INTRODUÇÃO

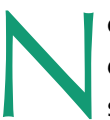
o âmbito da saúde mental a evolução histórica do processo assistencial vem sofrendo modificações em todos os aspectos, onde o modelo de cuidado médico hospitalocentrico busca ser superado para assumir um cuidado centrado na pessoa, família e comunidade. Processo que compreende a intersetorialidade e transdisciplinaridade como pontos fortes na busca da desinstitucionalização e do cuidado holístico ao indivíduo ${ }^{(1)}$.
Impulsiona-se no Brasil no final da década de 70 o movimento da reforma psiquiátrica brasileira, priorizando ações preventivas, de tratamento, promoção e reabilitação. Com vistas na desospitalização, as ações foram debatidas a fim de lançar uma assistência resolutiva para detectar, acolher e tratar integralmente os indivíduos com transtornos mentais de forma precoce, evitando internações desnecessárias ${ }^{(2)}$.

Assim, tendo em vista que a Atenção Primária à Saúde (APS) se caracteriza como a base de um sistema de saúde, além de ser a ordenadora das Redes de Atenção à Saúde (RAS), constitui-se como um nível de atenção essencial no processo de cuidado à saúde mental. Desse modo, estratégias de cuidados, como o acolhimento e o vínculo, devem ser utilizados pelos profissionais na perspectiva de um tratamento humanizado, adequado e fortalecido ${ }^{(3)}$. 
Nesse sentido, a Estratégia Saúde da Família (ESF) atua de forma conjunta com os demais serviços de outros níveis assistenciais, a fim de melhorar a resolutividade das necessidades encontradas nesta população, através da referência e contra referência. As ações de saúde mental devem ocorrer transcendendo o modelo tradicional, com o rompimento de estigmas que envolvem a saúde-doença por meio de um cuidado multiprofissional e interdisciplinar, com foco e aproximação do paciente, família e comunidade, a fim de atender suas reais necessidades ${ }^{(3)}$.

As ações desenvolvidas pelos profissionais de enfermagem na ESF no trabalho em saúde mental são concebidas na realidade local e contemplam o atendimento preventivo e acolhedor, propiciando uma assistência holística e humanizada, exercendo, portanto, um papel fundamental nessas circunstâncias. As ações devem ser baseadas nas diretrizes previamente estabelecidas pela Política Nacional de Saúde Mental, que propõe a mudança e tentativa de transformação do modelo tradicional psiquiátrico em uma assistência que inclua práticas que realize a inserção do indivíduo com transtorno psíquico na sociedade ${ }^{(4)}$.

O enfermeiro trabalha na perspectiva de criar laços entre o profissional, paciente e comunidade direcionando as intervenções de forma diferenciada a fim de trabalhar contrariamente ao modo asilar. Cabe ao enfermeiro desenvolver estratégias que reinsira os usuários na sociedade, onde junto a família irão se adaptar a comunidade. As práticas em saúde mental incluem o acolhimento, dialogo e a busca da melhor abordagem ao transtorno, com plano de assistência voltado a terapias em grupos, escuta e visitas domiciliares ${ }^{(5)}$.

Diante disso, questiona-se: quais as práticas em saúde mental desenvolvidas por enfermeiros da ESF? Com isso, o estudo tem como objetivo compreender as práticas de cuidado em saúde mental desenvolvidas por enfermeiros no âmbito da Estratégia Saúde da Família.

\section{MÉTODO}

Trata-se de uma pesquisa exploratória, de abordagem qualitativa, realizada em um município localizado na região Centro-Sul do Estado do Ceará em 2017. No âmbito da Atenção Primária à Saúde conta com 29 Equipes da Estratégia Saúde da Família, sendo 12 unidades na zona urbana e 17 na zona rural.

Participaram do estudo 10 enfermeiros que atuam nas Estratégias Saúde da Família da zona urbana. Utilizou-se como critério de inclusão: ser enfermeiro atuante na ESF no mínimo um mês, para que tenha condições de responder os questionamentos. Como critérios de exclusão considerou-se enfermeiros que estavam afastados do serviço, seja por estarem de férias ou de licença; e que não estiveram presentes no serviço após três visitas consecutivas da pesquisadora a unidade durante o período marcado para a coleta dos dados.

Para a coleta de dados foi elaborado um roteiro de entrevista semiestruturado. A entrevista foi composta por questões que englobam os aspectos sociodemográficos, profissionais e questões que nortearam a pesquisa. As entrevistas ocorreram em um único encontro, que foram previamente agendadas com os participantes da pesquisa e que durante sua execução foi utilizado um gravador portátil, com intuito de manter a fidedignidade das falas dos entrevistados, a partir do consentimento do participante do estudo.

Utilizou-se como método para análise dos dados a técnica de análise temática a partir das recomendações de Minayo $^{(6)}$, apresentando os resultados em categorias temáticas.

A pesquisa seguiu as recomendações estabelecidas pela Resolução 466/12 do Conselho Nacional de Saúde (CNS) do Ministério da Saúde e foi aprovado pelo Comitê de Ética em Pesquisa da Universidade Regional do Cariri (URCA), sob parecer No. 2314817.

\section{RESULTADOS E DISCUSSÃO}

Caracterização dos participantes

Participaram da pesquisa 10 enfermeiros atuantes nas ESF. Inicialmente, com vistas a caracterizar os participantes da pesquisa, foram identificados dados referentes as variáveis sexo, idade, tempo de formação, pós-graduação, tempo de atuação na atual unidade, o fato de possuir ou não capacitação e experiência na área de saúde mental.

A maioria dos participantes são do sexo feminino $(n=9)$, com idades entre 28 a 51 anos. A predominação da população feminina entre os profissionais de enfermagem que atuam na ESF é reconhecida, uma vez que a área da saúde tem uma tendência a feminização, porém a parcela masculina está aumentando significativamente $\mathrm{e}^{(7-8-9)}$.

Os resultados também se assemelham a uma pesquisa realizada por Faria, Acioli e Gallasch ${ }^{(10)}$, em que se evidenciou que a maioria dos enfermeiros que atuam na ESF são jovens acima dos 40 anos e com predominância do sexo feminino.

Quanto à capacitação a maioria referiu possuir pós-graduação $(n=9)$, dos quais sete possuem especialização em Saúde da Família e dois em Saúde Coletiva. Um estudo realizado em Minas Gerais revela a predominância das especializações em Saúde da Família na maioria dos profissionais enfermeiros ${ }^{(11)}$.

Foi identificado que somente dois dos participantes possuíam algum tipo de curso de capacitação em saúde mental, onde somente um destes descreveu o tipo de curso identificando-o como uma capacitação em matriciamento. É notória a necessidade de capacitação das pessoas para trabalhar com saúde mental, sendo imprescindível uma formação adequada tanto técnica como teórica desses profissionais para um cuidado adequado e um bom desempenho a esta clientela ${ }^{(12)}$.

Em relação à experiência em Saúde Mental, três dos entrevistados citaram ter alguma experiência na área, identificando a própria assistência realizada em seu serviço e o matriciamento, e apenas um 
dos entrevistados referiu ter possuído experiência na docência em Saúde Mental, enquanto que os demais evidenciaram não possuir qualquer experiência na área.

Compreensão dos enfermeiros sobre o cuidado em Saúde Mental na ESF

A maneira como distingue a concepção de saúde mental e a sua relação com a doença mental está ligada ao contexto o qual estamos inseridos, visto que as práticas nesse âmbito vêm sofrendo constantes transformações, principalmente no que se diz respeito à APS.

Frente ao material das entrevistas em relação à compreensão dos enfermeiros ao cuidado a saúde mental na ESF, estes divergem entre a compreensão acerca da saúde mental em si e suas ações na unidade em questão. Foram notórias as conceituações superficiais a respeito da saúde mental, haja vista que os profissionais consideraram algo amplo e complexo, mas não delimitam especificamente o que seria a saúde mental, como demonstra os relatos:

"Saúde mental [...] é um tema muito amplo, que vai desde uma coisa mais simples como uma insônia, uma ansiedade, que vai até coisas mais graves [...] como uma esquizofrenia ou uma coisa bem ampla [...]" (E 2)

"Saúde mental hoje está muito ampla, porque a gente houve falar demais que as pessoas estão com algum sofrimento psíquico, na verdade é isso, é algum sofrimento psíquico que esteja causando algum transtorno na vida dessa pessoa[...] " (E 10)

Percebe-se que a saúde mental é concebida como algo amplo, porém os entrevistados não delimitam a amplitude do termo. Referem que saúde está ligada com a ausência de doenças, sem contemplar os determinantes sociais dos termos e ainda atrelando a saúde mental aos aspectos psiquiátricos. Assim como é proposto o
“

Com a reforma psiquiátrica foi possível ter um olhar diferenciado sobre a doença mental, dando lugar a um cuidado humanizado as pessoas que passam por algum sofrimento psíquico. Os enfermeiros estão adotando uma postura diferente em relação a esse contexto, inserindo em suas práticas a responsabilidade do sujeito pela sua saúde, assim como a reabilitação e promoção do cuidado(13). conceito de saúde segundo a Organização Mundial de Saúde (OMS), o qual a postula como um estado de bem-estar não só físico como também biopsicossocial, superando o foco centrado na doença ${ }^{(13-14)}$.

Portanto, foi possível evidenciar que os enfermeiros devem ter um conhecimento ampliado de saúde-doença, principalmente no campo da saúde mental, onde os sinais e sintomas psíquicos devem sim ser identificados assim como as dificuldades enfrentadas cotidianamente por esses usuários e suas famílias, denotando-se a necessidade de um maior envolvimento social, além da interdisciplinaridade e ações territorializadas.

"[...] eu entendo a saúde mental como um cuidado mais especifico para um paciente que demanda de uma atenção meIhor, de uma equipe multiprofissional. Aquele paciente que a qualquer hora pode ter um surto dependendo da patologia a qual ele apresenta [...]." (E 1)

"A saúde mental não envolve só aqueles pacientes que tem algum problema, que são tidos como "loucos", envolve todo o processo de saúde do indivíduo." (E 5)

Com a reforma psiquiátrica foi possível ter um olhar diferenciado sobre a doença mental, dando lugar a um cuidado humanizado as pessoas que passam por algum sofrimento psíquico. Os enfermeiros estão adotando uma postura diferente em relação a esse contexto, inserindo em suas práticas a responsabilidade do sujeito pela sua saúde, assim como a reabilitação e promoção do cuidado(13).

Em relação ao trabalho que realizam na ESF, os relatos a seguir revelam que no município em questão a saúde mental ocupa lugar de destaque no âmbito da saúde mental, devido seu contexto histórico e sua estrutura de apoio. Contudo, o trabalho em saúde mental na ESF ainda se resume em renovação de receitas e encaminhamentos para outros serviços, 
evidenciando a escassez de ações a essa população nesse âmbito, o que pode fragmentar o cuidado desses indivíduos que embora sejam acompanhados pelas unidades especializadas, não deixam de pertencer a uma comunidade.

"[...]. Aqui geralmente os pacientes vêm fazer renovação de receita, onde muitas vezes mandam um familiar. [...] então o contato que a gente tem com esses pacientes é quase nada. Aqui não tem matriciamento, então a gente não tem esse preparo todo, mais aprofundado, para saber lhe dar com esses transtornos." (E 6)

Os enfermeiros sentem dificuldades de acolher as pessoas com transtornos mentais e acabam por focalizar no cuidado ao corpo, prejudicando as questões subjetivas que os envolvem. Outra dificuldade encontrada é a identificação do sofrimento psíquico nesse nível assistencial. As equipes que se inserem na ESF não têm experiência ou formação no que tange a saúde mental, somente em alguns momentos na formação superior, notando-se que as demandas requerem uma prática nessa área, que se mostram desfavoráveis para promoção da saúde nesse cenário ${ }^{(14,15)}$.

É necessária a capacitação dos enfermeiros para atender aos usuários com algum tipo de sofrimento psíquico, considerando as novas formas de saberes são exigidas na construção de um cuidar alicerçado em ações resolutivas e com quebra de paradigmas que historicamente estão enraizados no cuidado em saúde mental ${ }^{(16)}$.

Práticas do enfermeiro no cuidado à Saúde Mental na ESF

Nota-se que os profissionais de enfermagem quando questionados sobre a assistência prestada aos indivíduos com algum sofrimento mental demostraram notória preocupação quanto ao cuidado a problemas mais graves e a necessidade de encaminhamentos para serviços espe- cializados. Com isso, as ações na APS a este público são limitadas e faltam ações de promoção e prevenção, visto que a ESF enfatiza essa assistência.

"... os pacientes de saúde mental [...] estão vindo diretamente para o médico. Os casos que eu ainda peguei, foram os casos de crianças com alguma alteração, agitação, a mãe muito nervosa, muito agressiva, até mesmo com a criança, que a gente teve que estar referenciando para o CAPS [...]" (E 7)

Os pacientes com transtornos psíquicos quando atendidos na ESF tem o cuidado pautado na medicalização e fragmentação do sujeito, com uma série de obstáculos para um processo de mudança da assistência, tornando difíceis as práticas reabilitatórias, conduzindo cada vez mais o médico como centro do cuidado e valorizando a assistência especializada. Para que haja mudança nesse cenário os profissionais de enfermagem, assim como todos os demais profissionais da APS, devem desenvolver habilidades para acolher, escutar essas pessoas e incluí-las em seus cuidados cotidianos, promovendo os vínculos afetivos e de compromissos entre o paciente, profissional e família ${ }^{(17-18)}$.

Quando investigados sobre as estratégias que melhor respondiam a demanda de saúde mental pelos enfermeiros, verificou-se que há necessidade de ações de cuidado e ausência de ações características para esses usuários, ou ainda, diante das queixas apresentadas pelo paciente as ações passam a ser fundamentadas na conversa, orientação e o encaminhamento para outro serviço, conforme relatos a seguir:

“[...] não há nada que a gente faça que seja voltada a saúde mental, infelizmente [...] Estratégias especificas não existem. Chega um paciente com algum sintoma e agente encaminha para o médico daqui, ou psicólogo, ou CAPS e pronto! " (E 10)

"Então, se for alguma coisa relacionada a saúde mental eu procuro conversar, entender as razoes porque ele está daquela forma, se é familiar, algo amoroso ou relacionado a trabalho[...]. Encaminho para o médico, e se eu não tiver médico na unidade é que eu encaminho para o CAPS [...]" (E 3)

Outros profissionais relatam incluir em suas atividades, outra forma de cuidado, que é denominado acolhimento:

“[...] a gente faz o acolhimento, faz a escuta, tenta fazer uma escuta qualificada realmente, buscar quais são as principais queixas, os sintomas [...] faz toda a anotação tanto no prontuário do paciente como na ficha de encaminhamento de referência para o CAPS, ai faz o encaminhamento. " (E 6)

"O acolhimento da forma adequada é o que está fazendo com que esses pacientes se sintam mais à vontade para procurar a unidade [...]. Por enquanto só isso, já que eu não tenho nada planejado[...]." (E 7)

Estudo realizado em uma unidade básica de saúde do município de Campinas, São Paulo ${ }^{(19)}$, também aponta que os profissionais de enfermagem mantem a prática do encaminhamento dos usuários com transtornos mentais para uma equipe especializada, contudo estes demonstram um interesse em melhorar suas ações no que diz respeito ao acolhimento com uma assistência mais humanizada, com a capacidade de identificar o que o paciente está sentindo, diferenciado da assistência tecnicistas ${ }^{(19)}$.

Se faz necessário o estabelecimento de vínculo efetivo entre o usuário e o profissional, baseado em aceitação, confiança e receptividade, para contribuir diretamente no desempenho das ações, 
otimizando a assistência e proporcionando o acesso desses indivíduos ao serviço. Todos esses elementos estão incluídos no acolhimento, que é de responsabilidade profissional, assumindo uma postura de respeito as necessidades de saúde do usuário, além de fornecer maior segurança aos mesmos para que possam procurar o serviço de saúde ${ }^{(19)}$.

Dentro da perspectiva do cuidado de enfermagem aos pacientes com sofrimento mental é preciso que haja uma atuação ativa que contribua para o enfrentamento dos problemas e dificuldades da assistência a esse público e seus familiares. É evidente o despreparo em relação a sensibilização profissional e a necessidade de treinamento com um olhar voltado para a valorização do acolhimento, baseada em escuta e orientação adequada para uma assistência qualificada.

\section{CONCLUSÃO}

No tocante as ações realizadas nos serviços, é apontado o acolhimento, a escuta, a preocupação com o retorno do usuário as unidades, apresentando um impacto positivo na atenção a esses clientes.
Foi perceptível que muitas ações são destinadas ao referenciamento dos pacientes para os serviços especializados.

Evidenciou-se ainda que os enfermeiros devem adotar um posicionamento em relação ao trabalho em saúde mental com vista a enfrentar as questões que limitam seu trabalho cotidiano e superar o modelo tradicional de tratar esses indivíduos, colocando em suas ações a busca constante pelo conhecimento e aprimoramento cientifico, ações terapêuticas, reinserção do indivíduo na comunidade e empatia para lidar com o sofrimento do outro.

\section{Referências}

1. Amarante $P$, Nunes $M O$. A reforma psiquiátrica no SUS e a luta por uma sociedade sem manicômios. Ciênc. saúde coletiva. 2018; 23(6): 2067-2074. DOI: http://dx.doi.org/10.1590/141381232018236.07082018

2. Nascimento KC, Kolhs M, Mella S, Berra E, Olschowsky A, Guimarães $A N$. 0 desafio familiar no cuidado às pessoas acometidas por transtorno mental. Rev. enferm. UFPE on line. 2016; 10(3): 940-8. Available from: https://periodicos.ufpe.br/revistas/revistaenfermagem/article/viewFile/11044/12449.

3. Wenceslau LD, Ortega F. Saúde mental na atenção primária e Saúde Mental Global: perspectivas internacionais e cenário brasileiro. Interface (Botucatu, Online). 2015; 19(55):1121-1132. DOI: http:// dx.doi.org/10.1590/1807-57622014.1152.

4. Merces AMF, Souza BML, Silva TL, Moura Silva TT, Cavalcanti AMTS. Práticas de enfermagem em saúde mental na estratégia de saúde da família: revisão integrativa. Cogitare enferm [Internet]. 2015; 20(2):417-425. Available from: https://www.redalyc.org/articulo. oa? id=483647679023.

5. Nascimento MGG. Nursing work process in the promotion of mental health: reflective analysis. Rev. enferm. Cent.-Oeste Min 2017; 7/2097. DOI: http://dx.doi.org/10.19175/recom.v7i0.2097.

6. Minayo MCS. 0 Desafio do Conhecimento: Pesquisa qualitativa em saúde. $13^{\circ}$ ed. Editora Hucitec. São Paulo. 2013.

7. Gonçalves $C R$, Cruz MT, Oliveira MP, Morais AJD, Moreira KS, Rodrigues CAQ, Leite MTS. Human resources: critical factor for primary health networks. Saúde Debate (online). 2014; 38(100): 26-34. Available from: http://www.scielo.br/pdf/sdeb/v38n100/ 0103-1104-sdeb-38-100-0026.pdf

8. Santos ÁS, Silveira RE, Mendonça FTNF, Buso ALZ, Ferreira-Silva $S G$, Silva DD. Profile of managers of primary health care of a city of Minas Gerais, Brazil. Rev. enferm. atenção saúde. 2016; 5(1): 3-11. Available from: http://seer.uftm.edu.br/revistaeletronica/index.php/ enfer/article/view/1392/pdf_1

9. Machado $M H$, Aguiar-Filho W, Lacerda WF, Oliveira E, Lemos W, Wermelinger $M$, Vieira $M$, Santos $M R$, Souza-Junior $P B$, Justino $E$, Barbosa C. Caracteristicas gerais da enfermagem: o perfil sócio demográfico. Enferm. foco (Brasília). 2016; 7(ESP): 9-14. Available from: http://revista.cofen.gov.br/index.php/enfermagem/article/ view/686/296

10. Faria MGA, Acioli S, Gallasch CH. Perfil de enfermeiros flu- minenses da estratégia de saúde da família participantes de um curso de especialização. Enferm. foco. 2016; 7(1):52-55. Available from: http://revista.cofen.gov.br/index.php/enfermagem/article/ view/667/285

11. Marinho LM, Caetite LC, Lima CA, Ribeiro CDAL. Atributos da Atenção Primária: perspectiva e perfil de enfermeiros da Estratégia Saúde da Família. Revista Norte Mineira de Enfermagem. 2015; 4(2): 04-18. Available from: http://www.renome.unimontes.br/index.php/renome/article/view/90/130.

12. Silva SP, Oliveira AL, Kamimura QP. Capacitação em saúde mental: Entre a realidade e as ofertas do ministério da saúde. Revista Eletrônica Sistemas \& Gestão. 2014; 9(3): 406-416. DOI: https://doi. org/10.7177/sg.2014.V9.N3.A16.

13. Santos $A B$, Silva GG, Pereira MER, Brito RS. Saúde mental, humanização e direitos humanos. Brazilian Journal of Mental Health. 2018; 10(25): 01-19. Available from: http://incubadora.periodicos. ufsc.br/index.php/cbsm/article/view/4990/5201.

14. Buss PM, Pellegrini-Filho A. A saúde e seus determinantes sociais. Physis: Rev. Saúde Coletiva. 2007; 17(1): 77-93. DOI: http:// dx.doi.org/10.1590/S0103-73312007000100006.

15. Souza J, Yamawaka de Almeida L, Villar-Luis MA, Nievas AF, Veloso TMC, Barbosa SP, Giacon BCC, Assad FB. Mental health in the Family Health Strategy as perceived by health professionals. Rev. bras. enferm. 2017. 70(5): 985-992. DOI: http://dx.doi. org/10.1590/0034-7167-2016-0492.

16. Costa TD. et al. Contribuindo para a educação permanente na saúde mental. Biológicas \& Saúde. 2017; 7(23): 9-15. DOI: https:// doi.org/10.25242/88687232017647.

17. Neves $H G$, Lucchese $R$, Munari DB. Saúde mental na atenção primária: necessária constituição de competências. Rev. bras. enferm. 2010; 63(4): 666-670. DOI: http://dx.doi.org/10.1590/S003471672010000400025 .

18. Silva-Filho JA, Bezerra AM. Acolhimento em Saúde Mental na Atenção Primária à Saúde: Revisão Integrativa. Id on Line Rev. Mult. Psic. 2018; 12(40): 613-627. D0I: https://doi.org/10.14295/idonline. v12i40.1138.

19. Sucigan DHI, Toledo VP, Garcia APRF. Acolhimento e saúde mental: Desafio profissional na estratégia saúde da família. Rev. Rene. 2012; 13(1): 2-10. DOI: http://dx.doi.org/10.15253/rev\%20rene. v13i1.3756. 